\title{
LETTER
}

\section{Managing bone health in women with breast cancer under adjuvant treatment with aromatase inhibitors: pretreatment bone mineral density is important}

\author{
Christos Markopoulos* \\ See related editorial by Van Poznak, http://breast-cancer-research.com/content/12/3/110, and related research by Markopoulos et al., \\ http://breast-cancer-research.com/content/12/2/R24
}

In a recent editorial, Dr Van Poznak addresses the important issue of managing bone health in breast cancer patients receiving adjuvant treatment with an aromatase inhibitor [1]. Based on the published results of three clinical trials using risedronate $[2,3]$ or ibandronate [4], it is concluded that oral bisphosphonates may be considered a supportive therapy to treat or prevent osteoporosis in breast cancer patients receiving aromatase inhibitor adjuvant treatment.

We would like to comment on the results of those trials summarised in Table 1 of the editorial [1], focusing on patients with normal bone mineral density (BMD) or mild osteopaenia before starting treatment.

All three trials have shown that patients with normal BMD before starting treatment with anastrozole are at very low risk to develop osteoporosis, having a change in BMD less than $-6 \%$ measured at 2 years in both the lumbar spine and hip, without any pharmacologic intervention apart from receiving vitamin $\mathrm{D}$ and calcium supplements [2-4]. The risk of osteoporosis is minimal and only a few patients might move to the osteopaenic region.

Considering the grey zone of treatment decisions in the management of patients with osteopaenia, we agree those trials provide very useful clinical data on the need for pharmacologic intervention and the effectiveness of oral bisphosphonates. Even though the BMD loss is less than $-4 \%$ in both the lumbar spine and hip at 2 years under anastrozole treatment, the addition of oral bisphosphonates results in a change of up to $+5.7 \%$ in BMD that is statistically significant in all three trials - preventing

\footnotetext{
*Correspondence: cmarkop@hol.gr

Department of Surgery, Athens University Medical School, Greece, 8 lassiou Street, 11521 Athens, Greece
}

effectively the development of osteoporosis. Furthermore, the mixed models analysis of the randomised arms in the ARBI trial [3] examining the percentage change from baseline in hip and lumbar spine BMD values suggests that higher baseline BMD values correspond to smaller changes at both 12 and 24 months, whereas patients' smaller BMD values at baseline exhibited a higher trend of BMD increase. In other words, patients starting treatment with anastrozole are not all at the same risk for bone loss, and also they respond to a different extent to pharmacologic interventions pretreatment BMD estimation is an important factor in management decisions.

In conclusion, we estimate that all trials provide evidence supporting the use and effectiveness of oral bisphosphonates in patients already having BMD in the region of severe osteopaenia/osteoporosis when starting treatment with an aromatase inhibitor. On the contrary, for patients with normal BMD or mild osteopaenia at baseline, general preventive measures and BMD evaluation during follow-up visits seem to be adequate management since these patients are at low risk of developing severe osteopaenia or osteoporosis.

\section{Abbreviations \\ $\mathrm{BMD}$, bone mineral density.}

\section{Competing interests}

The author has received educational grants and lecture honoraria from AstraZeneca (London, UK), Novartis (Basel, Switzerland), and Pfizer Inc. (New York, USA).

\section{Published: 3 August 2010}

\section{References}

1. Van Poznak C: Managing bone mineral density with oral bisphosphonate therapy in women with breast cancer receiving adjuvant aromatase inhibition. Breast Cancer Res 2010, 12:110.

2. Van Poznak C, Hannon RA, Mackey JR, Campone M, Apffelstaedt JP, Clack G, Barlow D, Makris A, Eastell R: Prevention of aromatase inhibitor-induced 
bone loss using risedronate: the SABRE trial. J Clin Oncol 2010, 28:967-975

3. Markopoulos C, Tzoracoleftherakis E, Polychronis A, Venizelos B, Dafni U,

Xepapadakis G, Papadiamantis J, Zobolas V, Misitzis J, Kalogerakos K,

Sarantopoulou A, Siasos N, Koukouras D, Antonopoulou Z, Lazarou S, Gogas

$\mathrm{H}$ : Management of anastrozole-induced bone loss in breast cancer

patients with oral risedronate: results from the ARBI prospective clinical trial. Breast Cancer Res 2010, 12:R24.

4. Lester JE, Dodwell D, Purohit OP, Gutcher SA, Ellis SP, Thorpe R, Horsman JM, Brown JE, Hannon RA, Coleman RE: Prevention of anastrozole-induced bone loss with monthly oral ibandronate during adjuvant aromatase inhibitor therapy for breast cancer. Clin Cancer Res 2008, 14:6336-6342.

doi:10.1186/bcr2609

Cite this article as: Markopoulos C: Managing bone health in women with breast cancer under adjuvant treatment with aromatase inhibitors: pretreatment bone mineral density is important. Breast Cancer Research 2010, 12:403 